\title{
Bilateral Thalamic Infarction Following Transsphenoidal Surgery
}

\author{
Tejas Sankar, Jenny Souster, David E. Steinke
}

Can. J. Neurol. Sci. 2008; 35: 522-525

The transsphenoidal approach is widely employed for access to surgical lesions of the sella. Following the introduction of the operating microscope to the procedure by Hardy, ${ }^{1}$ it has become a safe and well-established operation, as demonstrated by the low morbidity and mortality in several large case series over the past four decades..$^{2-6}$ Nevertheless, rare but serious complications, most notably injury to the intracavernous carotid $\operatorname{artery}^{7,8}$ and post-operative meningitis, ${ }^{9}$ do occur and have been well-documented.

In this article we present, to our knowledge, the first reported case of bilateral thalamic infarction occurring as a serious complication of transsphenoidal surgery for resection of a pituitary adenoma. We also briefly review the pathogenesis of bilateral thalamic infarctions, and discuss the intraoperative factors unique to this case and to the transsphenoidal approach which may have contributed to this unusual complication.

\section{CASe Description}

A 50-year-old Caucasian woman with medically-controlled hypertension and a 20 pack-year smoking history originally presented in 1997 with worsening bitemporal hemianopsia, intermittent headache, and amenorrhea. Magnetic resonance imaging (MRI) revealed a large sellar mass with suprasellar extension. She subsequently underwent a subtotal transsphenoidal resection of the mass, which was classified as a null cell pituitary macroadenoma on pathological assessment. Postoperative recovery was uneventful and there were no permanent visual field deficits. The patient showed neither symptoms of adrenal insufficiency nor abnormal serum cortisol levels during follow-up. She was followed with serial MRI examinations, which remained unchanged until early 2005, when the tumor recurred. The MRI at that time demonstrated cystic suprasellar extension, close apposition to the optic apparatus, and invasion of the right cavernous sinus by the tumor (Figure 1). Although preoperative visual field testing was normal, the rapid tumor progression was thought to pose an impending threat to vision. Given a normal serum prolactin level $(23.8 \mu \mathrm{g} / \mathrm{l})$, medical therapy was not considered to be an option. A decision was made to perform repeat operative debulking of the tumor. Routine pre-operative labwork was within normal limits, including a serum hormone profile which showed normal levels of AM cortisol (300 nmol/l), thyroid stimulating-hormone (1.57 $\mathrm{mIU} / \mathrm{l})$, free T4 (17 pmol/l), and insulin-like growth factor (100 $\mathrm{ng} / \mathrm{ml})$. Electrocardiogram and chest-X-ray were normal.

Immediately prior to surgery the patient was given $100 \mathrm{mg}$ of Solucortef (hydrocortisone sodium) intravenously. A standard transsphenoidal approach under general endotracheal anaesthesia was employed to attack the tumor. The opening was performed by the Otolaryngology service as per the usual arrangement at our facility, and the previously reconstructed sellar floor was taken down without difficulty. A large superficial cystic portion of the tumor was immediately visualized and drained with microsuction. Subsequently, the remaining tumor, which was noted to be particularly firm, was debulked with a combination of curettage and suction. Intraoperative blood loss was minimal. Three transient drops of blood pressure occurred

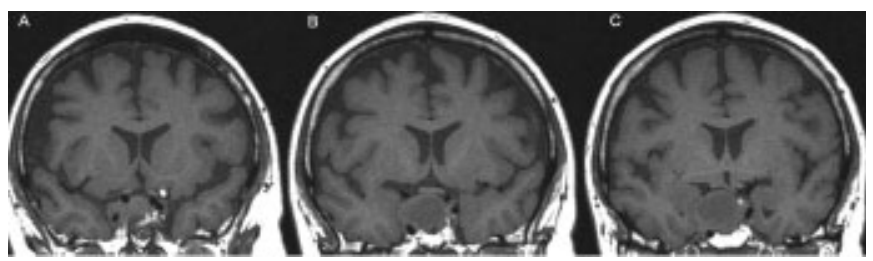

Figure 1. Coronal T1-weighted MRI showing a large, recurrent, null cell pituitary adenoma. (A) Invasion of the right cavernous sinus with encasement of the cavernous internal carotid artery. (B) Suprasellar extension with a large cystic component in close proximity to the optic apparatus. (C) Displacement of the pituitary stalk by tumor cyst.

during the procedure, each characterized by a systolic blood pressure of approximately $85 \mathrm{mmHg}$ and a diastolic pressure of $40 \mathrm{mmHg}$. These episodes were corrected with $10 \mathrm{mg}$ doses of intravenous ephedrine and lasted less than five minutes each. After the attending surgeon deemed the resection to be sufficient,

From the Division of Neurosurgery, Department of Surgery, University of Alberta, Edmonton, Alberta, Canada.

Received October 11, 2007. Final Revisions Submitted March 19, 2008. Correspondence to: David E. Steinke, 2D1.02 Walter C. Mackenzie Health Sciences Centre, University of Alberta, Edmonton, Alberta, T6G 2B7, Canada. 


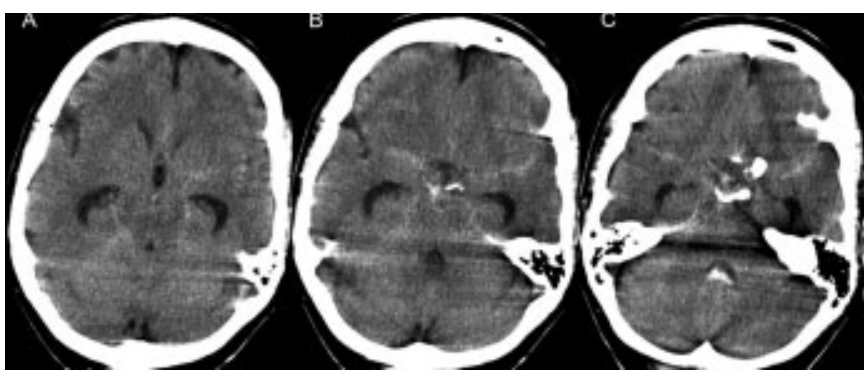

Figure 2. Immediate post-operative CT scan showing (A) ventriculomegaly with obvious dilation of the temporal horns of the lateral ventricles; (B) small volume subarachnoid hemorrhage visible in the basal cisterns; and $(C)$ small volume 4th ventricular hemorrhage.

the operative field was inspected and a small cerebrospinal fluid leak was observed from the left upper portion of the sella. Subcutaneous fat and Tisseal were used to repair the leak. The patient was extubated and taken to the recovery room in drowsy but stable condition.

In the recovery room, the patient remained drowsy with a Glasgow Coma Score 11-12, and exhibited fluctuating pupillary size and reaction to light. She was hemodynamically stable. An urgent head CT demonstrated a small amount of subarachnoid hemorrhage in the basal cisterns, some blood in the 4th ventricle, mild ventriculomegaly, and normal thalami (Figure 2). Lab work was normal. Subsequently the patient became more arousable

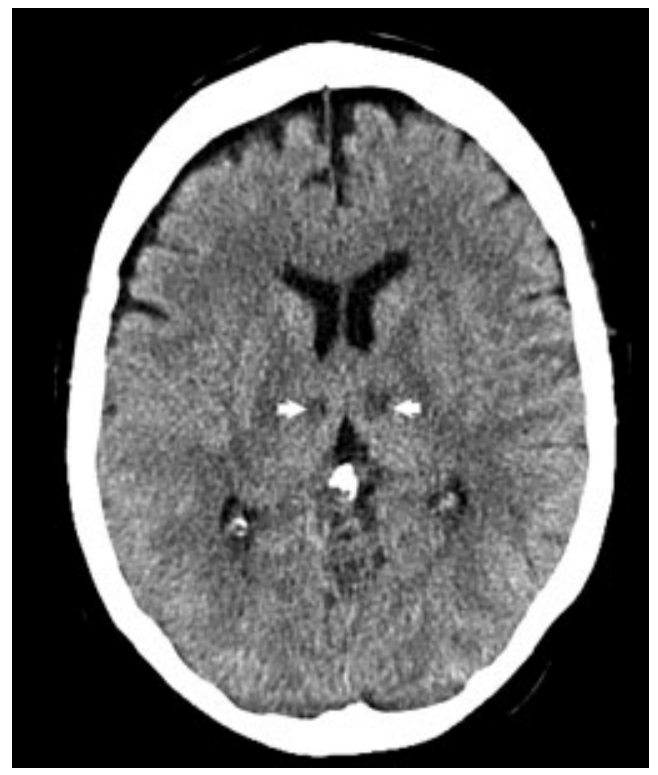

Figure 3. CT scan at 48 hours post-operation demonstrating bilateral thalamic hypodensities consistent with bilateral paramedian thalamic infarction, indicated by white arrows. and obeyed commands with all four extremities, so she was taken to the Neurosurgical Observation Unit. Her clinical picture was attributed to the intraventricular hemorrhage and associated obstructive hydrocephalus.

Over the next 24 hours, the patient's level of consciousness improved steadily but she remained disoriented. Her examination demonstrated anterograde amnesia, fluctuating anisocoria, and skew deviation. A CT scan at 48 hours postoperation demonstrated a reduction in subarachnoid blood and ventricular size, but also revealed bilateral medial thalamic hypodensities consistent with infarction (Figure 3). Subsequent FLAIR MRI confirmed bilateral paramedian thalamic infarcts (Figure 4).

The patient underwent formal cognitive assessment by the stroke rehabilitation team and was found to exhibit several features of bilateral paramedian thalamic infarction syndrome including inattention, severe impairment of short- and long-term memory, confabulation, disinhibition, and poor judgment.

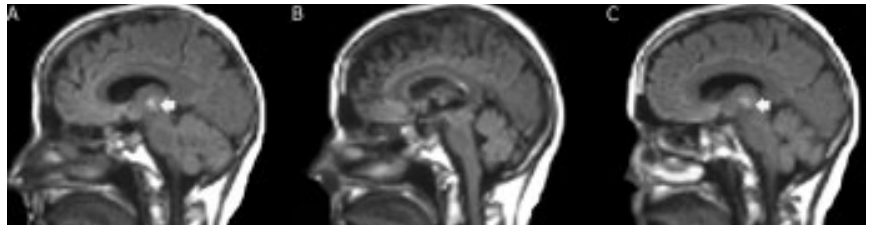

Figure 4. Postoperative sagittal FLAIR MRI confirming completed bilateral paramedian thalamic infarcts. (A) Section through left thalamus with obvious area of infarction (white arrow). (B) Section through 3rd ventricle. (C) Section through right thalamus, again showing an infarct (white arrow).

Pupillary abnormalities and skew deviation resolved by postoperative day ten, and the patient was discharged home with a plan for intensive outpatient rehabilitation. On follow-up at six months she was showing some improvement in cognitive function, but remained socially disinhibited and unable to work. Coronal MRI taken at that time showed satisfactory resection of the tumor with obliteration of the previous cystic component and decompression of the optic apparatus (Figure 5). Follow-up serum hormone profile was normal, with an AM cortisol level of $290 \mathrm{nmol} / 1$ essentially unchanged from prior to surgery.

\section{Discussion}

Bilateral thalamic infarction is rare, comprising less than $1 \%$ of all stroke cases. ${ }^{10}$ The most common subtype affects the paramedian thalamic territory, ${ }^{11}$ and produces a variable neurological syndrome that typically includes depressed or fluctuating level of consciousness, Korsakoff-type amnesia, and skew deviation, ${ }^{12}$ all of which were observed in our patient. Risk factors for bilateral paramedian thalamic infarction include those commonly associated with ischemic stroke, ${ }^{11}$ although shearing 


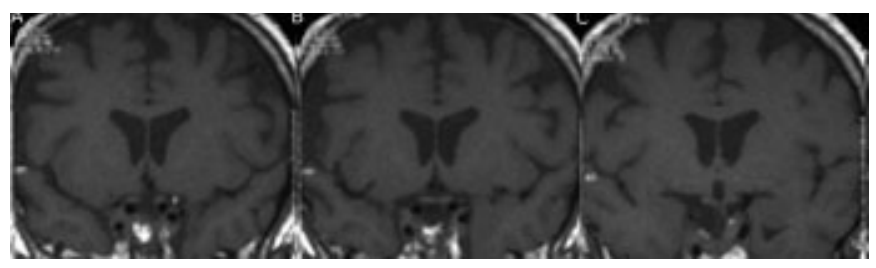

Figure 5. Postoperative coronal T1-weighted MRI demonstrating extent of tumor resection. Sections in $A, B$, and $C$ correspond approximately to sections $A, B$, and $C$ in Figure 1. (A) Extensive midline resection of tumor with persistent encasement of the right cavernous carotid artery by residual tumor. (B) Complete absence of previous cystic component and optic apparatus now free of apposing tumor. (C) Mild persistent displacement of pituitary stalk, but absence of previous tumor cyst.

trauma can also play a role. ${ }^{13}$ To our knowledge, our patient represents the first reported case in which bilateral paramedian thalamic infarction occurred as a direct complication of a transsphenoidal procedure.

Blood supply to the medial thalamus is usually provided by the paired paramedian thalamic perforating arteries, or arteries of Percheron, which arise from the P1 segments of each posterior cerebral artery (PCA). Percheron also described a variation of this vascular pattern in which an unpaired perforating artery arises from the P1 segment on one side to supply the medial thalamus bilaterally (Figure 6). ${ }^{14}$ Bilateral paramedian thalamic infarction is thought to occur when such an unpaired artery of Percheron is occluded acutely ${ }^{15-20}$ which we believe to be the case in our patient. To date there has been only one case report of bilateral thalamic infarction in which the presumed variant artery was demonstrated radiographically on digital subtraction angiograms. ${ }^{11}$ In that case, angiography appears to have been ordered purely on academic grounds. In general, angiograms are not indicated for bilateral paramedian thalamic infarction because non-visualization of the unpaired artery of Percheron neither excludes its patency nor confirms its occlusion. ${ }^{18}$ In keeping with the prevailing recommendations in the literature, we chose to spare our patient the risks of conventional angiography.

While bilateral thalamic infarction following transsphenoidal surgery for pituitary adenoma has not been documented previously, cases of unilateral thalamic infarction have been reported. $5,21,22$ These cases point to key intraoperative factors which may also have contributed to the unique complication in the present case. Reddy et al, ${ }^{5}$ in a review of 200 transsphenoidal procedures over 11 years, had one case of left medial thalamic infarction occurring in the immediate postoperative period. As in our patient, the pituitary adenoma was recurrent, large, and nonsecretory. The tumor's consistency was unusually firm and required pituitary rongeurs for satisfactory resection. In an audit study of 126 transsphenoidal procedures, Sudhakar et $\mathrm{al}^{21}$ reported a single case of post-operative thalamic infarction accompanied, as in our case, by intraoperative CSF leak. Finally, Kuroyanagi et $\mathrm{al}^{22}$ reported an unusual case of a patient who underwent surgery for a large, non-functional pituitary adenoma

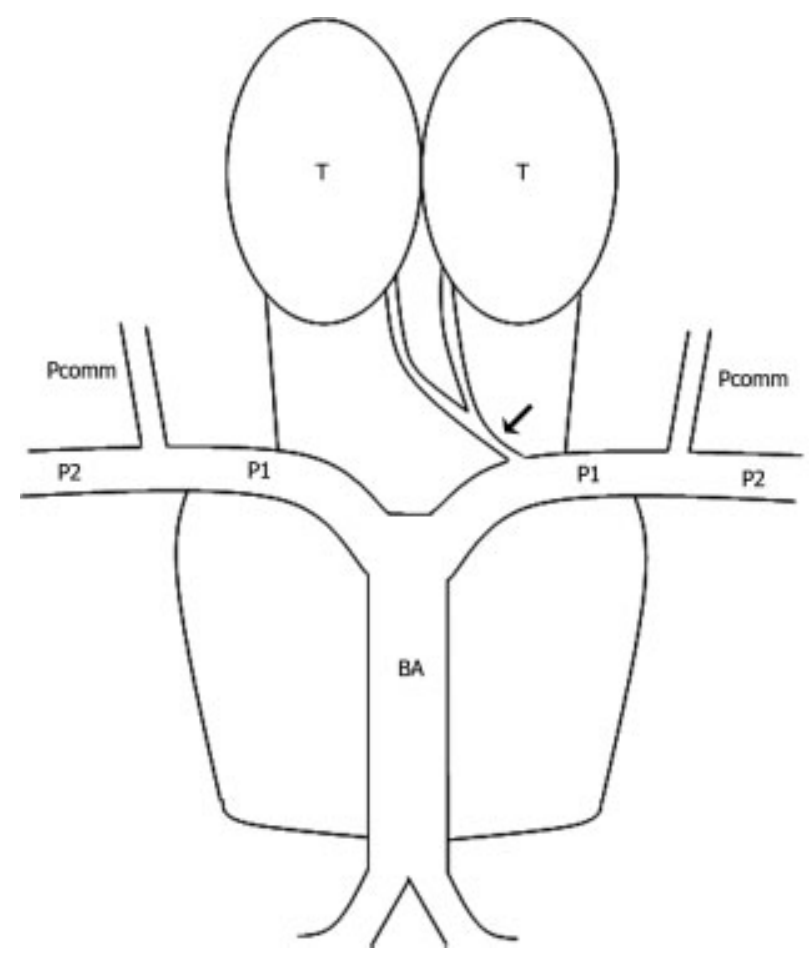

Figure 6. Schematic diagram of an unpaired artery of Percheron (black arrow) arising from the P1 segment of the posterior cerebral artery on one side and dividing to supply the medial thalami bilaterally. In our patient, the capsule of the large pituitary tumor may have been adherent to this artery, leading to its disruption or occlusion during surgical debulking with subsequent bilateral paramedian thalamic infarction. $T=$ thalamus, $B A=$ basilar artery, Pcomm = posterior communicating artery, $P 1, P 2=$ first and second segments of the posterior cerebral artery.

and developed a left medial thalamic infarction. In that case the initial infarction was associated, as in our patient, with subarachnoid hemorrhage, although this was followed by multiple unexplained episodes of brainstem hemorrhage within the first postoperative week.

Three mechanisms, acting alone or in combination, likely contributed to bilateral paramedian thalamic infarction in our patient. First, indirect damage to the thalamoperforating arteries, and in particular the artery of Percheron, probably occurred intraoperatively. In large tumors with suprasellar extension, branches of the PCA may be adherent to the tumor capsule.22 When the suprasellar portion descends into the sella as the resection proceeds, there may be tension on these vessels with their resultant disruption or occlusion. This tension may also be exacerbated by aggressive manipulation or the tumor, ${ }^{5}$ and we speculate in our case that the rapid decompression of a large cystic component of the tumor during the procedure could have placed the artery of Percheron under undue mechanical stress. A second contributory mechanism is thalamoperforator vasospasm. In our case an intraoperative CSF leak allowed for blood to enter the subarachnoid space, possibly causing selective spasm of the 
small caliber artery of Percheron. Of course, without postoperative angiography this is purely speculative. Furthermore, thalamic infarction in this case developed within 48 hours of surgery - much more rapidly than the ischemia seen following vasospasm secondary to subarachnoid hemorrhage. A third contributing factor may have been transient intraoperative hypotension, which could have further limited thalamic perfusion through an already disrupted or spastic artery of Percheron. Hypotension during pituitary surgery is frequently related to hypopituitarism and accompanying cortisol deficiency; however, a normal preoperative serum cortisol level and perioperative administration of intravenous corticosteroids make this an unlikely mechanism in our patient. We suspect instead that she may merely have had exaggerated vasomotor sensitivity to anaesthestic agents or relative hypovolemia during surgery.

The complication encountered in our patient, though unique, can serve as a reminder of the potential importance of several technical issues in transsphenoidal surgery. In particular, it highlights the fact that large or recurrent tumors with suprasellar extension are more likely to produce complications..$^{5,6,21,23}$ Such tumors are often of firm consistency, and surgical manipulation with traction on adjacent structures in such cases increases the likelihood of these complications. Gentle intraoperative maneuvering and avoidance of rapid tumor decompression are important to minimize capsule traction, as is avoidance, if at all possible, of an arachnoid breach with subsequent CSF leak. Though we did not employ an endoscope at any point during the procedure, the purely endoscopic transsphenoidal approach, by providing broad panoramic views of the surgical field and dynamic, magnified inspection of the tumor during resection, can facilitate these aims. ${ }^{24}$ Finally, meticulous avoidance of intraoperative hypotension remains an important surgical principle in preventing ischemic sequelae.

\section{Conclusions}

We report a case of bilateral paramedian thalamic infarction occurring as a complication of transsphenoidal surgery for recurrent pituitary adenoma. The case highlights the putative etiology of bilateral paramedian thalamic infarction due to an anatomic variant in thalamic blood supply, while serving as a reminder to neurosurgeons of the importance of meticulous surgical technique during the transsphenoidal approach.

\section{REFERENCES}

1. Hardy J. Transsphenoidal removal of pituitary adenomas. Union Med Can. 1962;91:933-45.

2. Hardy J. Transsphenoidal hypophysectomy. J Neurosurg. 1971;34: 582-94.

3. Wilson CB, Dempsey LC. Transsphenoidal microsurgical removal of 250 pituitary adenomas. J Neurosurg. 1978;48:13-22.

4. Kennedy DW, Cohn ES, Papel ID, Holliday MJ. Transsphenoidal approach to the sella: The Johns Hopkins experience. Laryngoscope. 1984;94:1066-74.

5. Reddy K, Fewer D, West M. Complications of the transsphenoidal approach to sellar lesions. Can J Neurol Sci. 1991;18:463-6.

6. Ciric I, Ragin A, Baumgartner C, Pierce D. Complications of transsphenoidal surgery: results of a national survey, review of the literature, and personal experience. Neurosurgery. 1997; $40: 225-36$
7. Paullus WS Jr., Norwood CW, Morgan HW. False aneurysm of the cavernous carotid artery and progressive external ophthalmoplegia after transsphenoidal hypophysectomy. Case report. J Neurosurg. 1979;51:707-9.

8. Cabezudo JM, Carrillo R, Vaquero J, Areitio E, Martinez R. Intracavernous aneurysm of the carotid artery following transsphenoidal surgery. Case report. J Neurosurg. 1981;54: 118-21.

9. Gransden WR, Wickstead M, Eykyn S. Meningitis after transsphenoidal excision of pituitary tumors. J Laryngol Otol. 1988;102:33-6.

10. Kumral E, Evyapan D, Balkir K, Kutluhan S. Bilateral thalamic infarction. Acta Neurol Scand. 2001;103:35-42.

11. Roitberg BZ, Tuccar E, Alp MS. Bilateral paramedian thalamic infarct in the presence of an unpaired thalamic perforating artery. Acta Neurochir (Wien). 2002;144:301-4.

12. Schmahmann JD. Vascular syndromes of the thalamus. Stroke. 2003;34:2264-78.

13. Barontini F, Maurri S. Isolated amnesia following a bilateral paramedian thalamic infarct: possible role of a whiplash injury. Acta Neurol (Napoli). 1992;14:90-102.

14. Percheron G. Les artères du thalamus humain: II Artères et territoires thalamiques paramédiens de l'artère basillaire communicante. Rev Neurol (Paris). 1976;132;309-24.

15. Biller J, Sand JJ, Corbett JJ, Adams HP Jr., Dunn V. Syndrome of the paramedian thalamic arteries: clinical and neuroimaging correlation. J Clin Neuroopthalmol. 1985;5:217-23.

16. Lepore FD, Gulli V, Miller DC. Neuro-ophthalmological findings with neuropathological correlation in bilateral thalamicmesenecephalic infarction. J Clin Neuroophthalmol. 1985;5: 224-8.

17. Castaigne P, Lhermitte F, Buge A, Escourolle R, Hauw JJ, LyonCaen O. Paramedian thalamic and midbrain infarcts: clinical and neuropathological study. Ann Neurol. 1985;10:127-48.

18. Matheus MG, Castillo M. Imaging of acute bilateral paramedian thalamic and mesencephalic infarcts. AJNR Am J Neuroradiol. $2003 ; 24: 2005-8$.

19. Raphaeli G, Liberman A, Gomori JM, Steiner I. Acute bilateral paramedian thalamic infarcts after occlusion of the artery of Percheron. Neurology. 2006;66:E7.

20. Giannopoulos S, Kostadina V, Selvi A, Nicolopoulos P, Krystis A. Bilateral paramedian thalamic infarcts. Ann Neurol. 2006; 63:1652.

21. Sudhakar N, Ray A, Vafidis JA. Complications after transsphenoidal surgery: our experience and a review of the literature. Br J Neurosurg. 2004; 18:507-12.

22. Kuroyanagi $\mathrm{T}$, Kobayashi $\mathrm{S}$, Takemae $\mathrm{T}$, Kobayashi $\mathrm{S}$. Subarachnoid hemorrhage, midbrain hemorrhage and thalamic infarction following transsphenoidal removal of a pituitary adenoma. A case report. Neurosurg Rev. 1994;17:161-5.

23. Black PMcL, Zervas NT, Candia GL. Incidence and management of complications of transsphenoidal operations for pituitary adenomas. Neurosurgery. 1987;20:920-4.

24. Jane JA Jr., Han J, Prevedello DM, Jagannathan J, Dumont AS, Laws ER Jr. Perspectives on endoscopic transsphenoidal surgery. Neurosurg Focus. 2005;19:E2. 